\title{
Coupled gas and ion transport in quadrupole interfaces
}

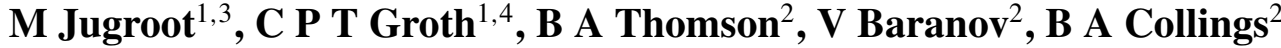 \\ and $\mathbf{J} \mathbf{B}$ French $^{2}$ \\ ${ }^{1}$ Institute for Aerospace Studies, University of Toronto, Ontario, M3H 5T6, Canada \\ ${ }^{2}$ MDS SCIEX, Concord, Ontario, L4K 4V8, Canada \\ E-mail: jugroot-m@rmc.ca and groth@utias.utoronto.ca
}

Received 12 October 2007

Published 4 January 2008

Online at stacks.iop.org/JPhysD/41/025205

\begin{abstract}
The transport of free ions through highly under-expanded jet flows of neutral gases and in the presence of applied electric fields is investigated by continuum-based (fluid) numerical simulations. In particular, numerical results are described which are relevant to ion flows occurring in quadrupole interfaces of mass spectrometer systems. A five-moment mathematical model and parallel multi-block numerical solution procedure are developed for predicting the ion transport. The model incorporates the effects of ion-neutral collision processes and is used in conjunction with a Navier-Stokes model and flow solver for the neutral gas to examine the key influences controlling the ion motion. The effects of the neutral gas flow, electric fields (both dc and rf) and flow field geometry on ion mobility are carefully assessed. The capability of controlling the charged particle motions through a combination of directed neutral flow and applied electric field is demonstrated for these high-speed, hypersonic, jet flows. The neutral dynamics is shown to have a strong influence on the ion transport whereas the electric field imparts a more gradual effect. The combined effect of the applied (dc and rf) electric field and neutral collision processes with the dilute neutral gas results in a strong tendency for ion focusing towards the axis of symmetry, with the overall efficiency governed by the mass-to-charge ratio.
\end{abstract}

(Some figures in this article are in colour only in the electronic version)

\section{Introduction}

The ability to accurately predict and thereby understand the often complex transport of charged particles through a background neutral gas subject to electro-magnetic forces is very important to furthering the understanding of many advanced technological processes including those associated with vapour deposition in material and semiconductor processing [1], electric space propulsion devices [2, 3], microsystems [4], plasma jets [5,6] as well as space plasmas [7]. The transport of ions through rapidly expanding and/or jet flows is also very important to the operation of mass spectrometers, such as liquid chromatography (LC)/mass spectrometry (MS) systems used extensively in the trace analysis of biological fluids for metabolites and natural

\footnotetext{
3 Assistant Professor, Royal Military College, Canada.

4 Associate Professor, University of Toronto, Canada.
}

biopolymers [8,9] and in drug design [10]. The LC/MS instruments make use of an atmospheric pressure ionization technique whereby sample ions are generated at atmospheric pressure from molecules which are contained in microdroplets and the resulting ions are then transported from the atmospheric pressure conditions into a high vacuum system for spectroscopic analysis. The performance of the mass spectrometers is highly dependent on the ion transport from the source region to the mass detectors. Gaining an improved understanding of ion-source flows and related transport phenomena is an active area of research.

This study is concerned with the modelling of the transport of free ions through highly under-expanded jet flows of neutral gases under the influence of applied electric fields produced in the interface regions of quadrupole mass spectrometer systems. Quadrupole interfaces generally consist of a radiofrequency (rf) quadrupole as shown in figure 1. A variety 


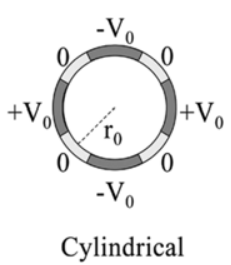

Cylindrical

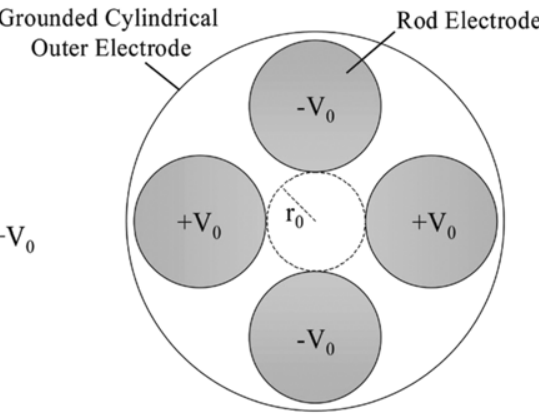

Traditional

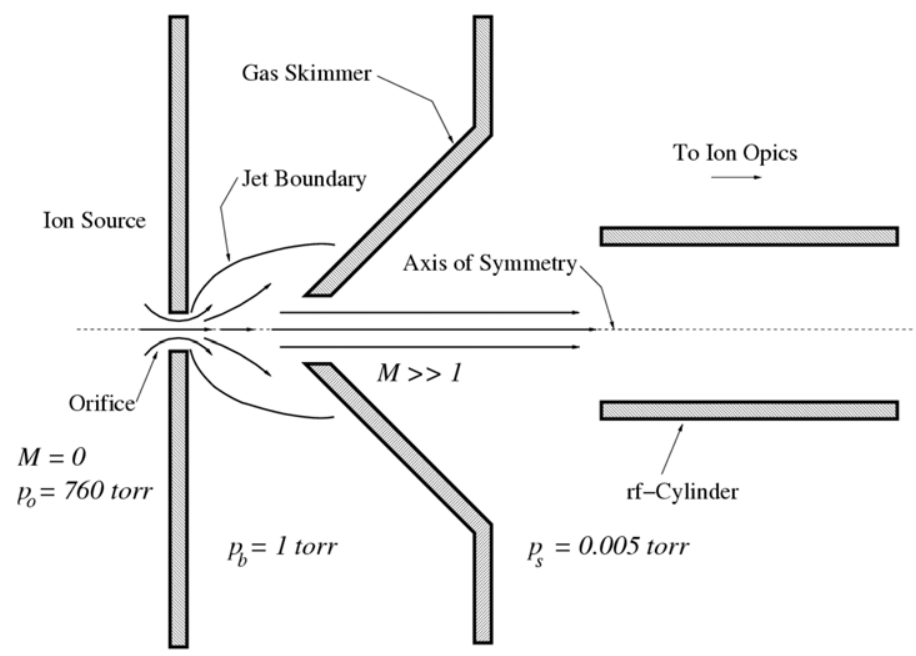

Figure 1. Schematic diagrams of (LHS) an rf cylinder showing the electrode and insulator strip geometry and the more traditional, equivalent, four-rod quadrupole configuration (from Prestage [11]) and (RHS) the quadrupole interface showing orifice, skimmer and rf cylinder.

of quadrupole configurations are possible. In this study, two different configurations are considered, namely, the traditional four-rod quadrupole configuration as shown in figure 1 as well as a novel configuration consisting of a single hollow cylinder composed of alternating electrode and insulator strips that produce the rf-quadrupole field [11], also shown in the figure. The region upstream of the orifice is at atmospheric pressure and, downstream of the quadrupole, a low pressure is maintained. Ions are transported by the neutral gas from the high-pressure reservoir through a small orifice and gas skimmer and then on through the rf quadrupole as shown in figure 1 .

A five-moment continuum-based (fluid) model and parallel multi-block numerical solution procedure are described for predicting the ion transport in the complete quadrupole interface. The five-moment model has been used successfully in previous studies of ion transport in MS systems by Jugroot et al $[12,13]$. It provides 'one-way' coupling between the ion and neutral flows by incorporating the effects of ion-neutral collision processes. It can also account for the effects of externally applied electric fields. A parallel explicit higher-order Godunov-type finite-volume scheme is used to solve the five-moment ion transport equations on a multi-block quadrilateral mesh. The proposed scheme determines the ion transport in the quadrupole interface, given a precomputed neutral gas flow and electric potential.

\section{Governing equations}

\subsection{Five-moment ion transport model}

The transport of a mixture of a neutral gas and free ions is considered. This mixture is not formally treated as a plasma, but rather as a source of positively charged ions, for it is assumed that there are no free electrons (plasmas comprise neutral particles, ions and electrons and the charged particles' transport is generally tightly coupled by electric/magnetic applied/induced fields). For the purpose of this study, it is also estimated and assumed that the ion number density is very low compared with that of the neutral gas such that the ions have a negligible effect on the neutral particles. Hence, the solution of the ion and neutral gas flows can be decoupled. The neutral gas flow field can be predetermined and then the prediction of the ion motion can be carried out using this calculated neutral gas solution. A five-moment closure continuum approximation is used to model the motion of the ions through the more dense neutral gas [14-17]. This mathematical description is a single-temperature, near-equilibrium model that can take into account the effects of ion-neutral collision processes and applied external electric and magnetic forces. Ion selfcollisions (ion-ion collision processes) are neglected in this approximation and hence the ion fluid stresses and heat flux are assumed to be unimportant.

The governing transport equations of the five-moment model reflect the conservation of mass, momentum and energy and can be written in the non-conservative coordinate-free form as

$$
\begin{aligned}
& \frac{\partial \rho_{\mathrm{i}}}{\partial t}+\nabla \cdot\left(\rho_{\mathrm{i}} \boldsymbol{v}_{\mathrm{i}}\right)=0 \\
& \frac{\partial \boldsymbol{v}_{\mathrm{i}}}{\partial t}+\left(\boldsymbol{v}_{\mathrm{i}} \cdot \boldsymbol{\nabla}\right) \boldsymbol{v}_{\mathrm{i}}+\frac{1}{\rho_{\mathrm{i}}} \boldsymbol{\nabla} p_{\mathrm{i}}=\frac{Q_{\mathrm{i}}}{m_{\mathrm{i}}} \boldsymbol{E}+\frac{Q_{\mathrm{i}}}{m_{\mathrm{i}}}\left(\boldsymbol{v}_{\mathrm{i}} \times \boldsymbol{B}\right) \\
& +\sum_{s} v_{\mathrm{is}}\left(\boldsymbol{v}_{s}-\boldsymbol{v}_{\mathrm{i}}\right) \\
& \frac{\partial p_{\mathrm{i}}}{\partial t}+\left(\boldsymbol{v}_{\mathrm{i}} \cdot \boldsymbol{\nabla}\right) p_{\mathrm{i}}+\gamma_{\mathrm{i}} p_{\mathrm{i}}\left(\boldsymbol{\nabla} \cdot \boldsymbol{v}_{\mathrm{i}}\right)=\frac{\delta p_{\mathrm{i}}}{\delta t},
\end{aligned}
$$

where $\rho_{\mathrm{i}}=m_{\mathrm{i}} n_{\mathrm{i}}$ is the ion mass density, $n_{\mathrm{i}}$ is the ion number density, $m_{\mathrm{i}}$ is the mass of the ion molecules, $\boldsymbol{v}_{\mathrm{i}}$ is the ion velocity, $p_{\mathrm{i}}=\rho_{\mathrm{i}} R_{\mathrm{i}} T_{\mathrm{i}}=n_{\mathrm{i}} k T_{\mathrm{i}}$ is the ion pressure, $T_{\mathrm{i}}$ is the ion temperature, $R_{\mathrm{i}}$ is the ion ideal gas constant, $k$ is the Boltzmann constant, $\gamma_{\mathrm{i}}$ is the ion specific heat ratio, $Q_{\mathrm{i}}$ is the ion particle charge and $\boldsymbol{E}$ and $\boldsymbol{B}$ are the external electric and magnetic fields. Ion-neutral collision processes are modelled here using a generalization of the so-called relaxation time or the BGK (Bhatnagar, Gross and Krook) approximation for 
the Boltzmann collision integral [14, 16-18]. In this modified relaxation-time approach, the relaxation time is actually not constant. Instead, the collision cross-section is assumed to be constant and the collision frequency is taken to be dependent on the local macroscopic solution quantities (i.e. number densities and temperatures). The influences of the ion-neutral collisional processes manifest themselves as source terms in the ion momentum and energy equations, equations (2) and (3), respectively. These terms involve a sum over all neutral gas species, $s$, and depend on the momentum exchange collision frequency, $v_{\mathrm{is}}$. The energy or pressure source term, $\delta p_{\mathrm{i}} / \delta t$, is given by

$\frac{\delta p_{\mathrm{i}}}{\delta t}=\sum_{s} \frac{\rho_{\mathrm{i}} m_{s} v_{\mathrm{i} s}}{\left(m_{\mathrm{i}}+m_{s}\right)^{2}}\left[2 k\left(T_{s}-T_{\mathrm{i}}\right)+\frac{2}{3} m_{s}\left|\boldsymbol{v}_{s}-\boldsymbol{v}_{\mathrm{i}}\right|^{2}\right]$,

where $T_{s}$ and $\boldsymbol{v}_{s}$ are the temperature and velocity of neutral species $s$ and $m_{s}$ is the mass of the neutral species molecules. The collision frequency, $v_{\text {is }}$, can be related to the product of the collision cross-section $\sigma_{\text {is }}$, neutral species number density, $n_{s}$, and relative speed of the colliding particles $g_{\text {is }}$. By utilizing the approximation that $g_{\mathrm{i} s}=\sqrt{\bar{v}_{\mathrm{i}}^{2}+\bar{v}_{s}^{2}}=$ $\sqrt{8 k T_{\mathrm{i}} / \pi m_{\mathrm{i}}+8 k T_{s} / \pi m_{s}}$, the following expression can be obtained and used to prescribe the ion-neutral collision frequency [17]:

$$
v_{\mathrm{i} s}=\sigma_{\mathrm{i} s} n_{s} g_{\mathrm{i} s}=\sigma_{\mathrm{i} s} n_{s} \sqrt{1+\frac{m_{s} T_{\mathrm{i}}}{m_{\mathrm{i}} T_{s}}} \sqrt{\frac{8 k T_{s}}{\pi m_{s}}} .
$$

The relaxation-time model is only an approximation to the Boltzmann collision integral and ignores the detailed nature of inter-particle interactions. Nevertheless, it retains many of the qualitative features of the true collision integral and is thought to be sufficient for this study of ion transport phenomena in mass spectrometer systems.

For a two-dimensional axisymmetric coordinate system, a neutral gas consisting of a single neutral species $s=n$ and negligible magnetic fields $(\boldsymbol{B}=0)$, the conservative form of the five-moment equations governing the ion transport can be summarized as follows:

$$
\frac{\partial \boldsymbol{U}_{\mathrm{i}}}{\partial t}+\frac{\partial \boldsymbol{F}_{\mathrm{i}}}{\partial z}+\frac{\partial \boldsymbol{G}_{\mathrm{i}}}{\partial r}=\boldsymbol{S}_{\mathrm{ai}}+\boldsymbol{S}_{\mathrm{ei}}+\boldsymbol{S}_{\mathrm{ci}}
$$

where $\boldsymbol{U}_{\mathrm{i}}$ is the conserved variable solution vector given by

$$
\boldsymbol{U}_{\mathrm{i}}=\left[\rho_{\mathrm{i}}, \quad \rho_{\mathrm{i}} u_{\mathrm{i}}, \quad \rho_{\mathrm{i}} v_{\mathrm{i}}, \quad \rho \epsilon_{\mathrm{i}}\right]^{\mathrm{T}},
$$

$z$ and $r$ are the axial and radial spatial coordinates of the axisymmetric frame, $u_{\mathrm{i}}$ and $v_{\mathrm{i}}$ are the ion axial and radial velocity components, $\epsilon_{\mathrm{i}}=p_{\mathrm{i}} /\left(\rho_{\mathrm{i}}\left(\gamma_{\mathrm{i}}-1\right)\right)+\left|\boldsymbol{v}_{\mathrm{i}}\right|^{2} / 2$ is the specific total energy, $\boldsymbol{F}_{\mathrm{i}}$ and $\boldsymbol{G}_{\mathrm{i}}$ are the axial- and radialdirection solution flux vectors given by

$$
\boldsymbol{F}_{\mathrm{i}}=\left[\begin{array}{c}
\rho_{\mathrm{i}} u_{\mathrm{i}} \\
\rho_{\mathrm{i}} u_{\mathrm{i}}^{2}+p_{\mathrm{i}} \\
\rho_{\mathrm{i}} u_{\mathrm{i}} v_{\mathrm{i}} \\
u_{\mathrm{i}}\left(\rho_{\mathrm{i}} \epsilon_{\mathrm{i}}+p_{\mathrm{i}}\right)
\end{array}\right], \quad \boldsymbol{G}_{\mathrm{i}}=\left[\begin{array}{c}
\rho_{\mathrm{i}} v_{\mathrm{i}} \\
\rho_{\mathrm{i}} u_{\mathrm{i}} v_{\mathrm{i}} \\
\rho_{\mathrm{i}} v_{\mathrm{i}}^{2}+p_{\mathrm{i}} \\
v_{\mathrm{i}}\left(\rho_{\mathrm{i}} \epsilon_{\mathrm{i}}+p_{\mathrm{i}}\right)
\end{array}\right]
$$

and $\boldsymbol{S}_{\mathrm{ai}}, \boldsymbol{S}_{\mathrm{ei}}$ and $\boldsymbol{S}_{\mathrm{ci}}$ are source vectors associated with the axisymmetric coordinate frame, electric fields and ion-neutral collision processes, respectively. The latter are given by

$$
\begin{gathered}
\boldsymbol{S}_{\mathrm{ai}}=-\frac{1}{r}\left[\begin{array}{c}
\rho_{\mathrm{i}} v_{\mathrm{i}} \\
\rho_{\mathrm{i}} u_{\mathrm{i}} v_{\mathrm{i}} \\
\rho_{\mathrm{i}} v_{\mathrm{i}}^{2} \\
v_{\mathrm{i}}\left(\rho_{\mathrm{i}} \epsilon_{\mathrm{i}}+p_{\mathrm{i}}\right)
\end{array}\right], \\
\boldsymbol{S}_{\mathrm{ei}}=\left[\begin{array}{c}
0 \\
\rho_{\mathrm{i}} \frac{Q_{\mathrm{i}}}{m_{\mathrm{i}}} E_{z} \\
\rho_{\mathrm{i}} \frac{Q_{\mathrm{i}}}{m_{\mathrm{i}}} E_{r} \\
Q_{\mathrm{i}} \\
\rho_{\mathrm{i}} \frac{\left.u_{\mathrm{i}} E_{z}+v_{\mathrm{i}} E_{r}\right)}{m_{\mathrm{i}}} \\
0 \\
\frac{\rho_{\mathrm{i}} m_{\mathrm{n}} v_{\mathrm{in}}}{m_{\mathrm{i}}+m_{\mathrm{n}}}\left(u_{\mathrm{n}}-u_{\mathrm{i}}\right) \\
\frac{\rho_{\mathrm{i}} m_{\mathrm{n}} v_{\mathrm{in}}}{m_{\mathrm{i}}+m_{\mathrm{n}}}\left(v_{\mathrm{n}}-v_{\mathrm{i}}\right) \\
\frac{\delta p_{\mathrm{i}}}{\delta t}+\frac{\rho_{\mathrm{i}} m_{\mathrm{n}} v_{\mathrm{in}}}{m_{\mathrm{i}}+m_{\mathrm{n}}}\left[u_{\mathrm{i}}\left(u_{\mathrm{n}}-u_{\mathrm{i}}\right)\right. \\
\left.+v_{\mathrm{i}}\left(v_{\mathrm{n}}-v_{\mathrm{i}}\right)\right]
\end{array}\right],
\end{gathered}
$$

where $E_{z}$ and $E_{r}$ are the axial and radial components of the electric field and $u_{\mathrm{n}}$ and $v_{\mathrm{n}}$ are the axial and radial components of the neutral gas velocity. Note the presence of Joule heating terms associated with the applied electric field in the source terms of the energy equation. The equation set of (6) is used here to predict ion particle motion through a high-speed neutral gas.

For low-speed ion flows through a single-species, stationary, neutral gas (neglecting inertial effects), the ion drift velocity can be directly related to the applied electric field. It follows from the ion momentum equation that

$$
\boldsymbol{v}_{\mathrm{i}}=\frac{Q_{\mathrm{i}}\left(m_{\mathrm{i}}+m_{\mathrm{n}}\right)}{m_{\mathrm{i}} m_{\mathrm{n}} v_{\mathrm{in}}} \boldsymbol{E}=\eta_{\mathrm{in}} \boldsymbol{E},
$$

where $\eta_{\text {in }}=Q_{\mathrm{i}}\left(m_{\mathrm{i}}+m_{\mathrm{n}}\right) / m_{\mathrm{i}} m_{\mathrm{n}} v_{\mathrm{in}}$ is the ion mobility. It is readily apparent from this expression that the BGK collision model leads to a linear relationship between the drift velocity and the electric field. Furthermore, the mobility derived from these expressions was found to be in very good agreement with empirical/experimental formulations [19].

\subsection{Navier-Stokes model for neutral flow}

A near-thermal-equilibrium continuum model is also used to describe the neutral gas flows. The neutral flow is assumed to be laminar and the gas is taken to be both calorically and thermally perfect and to obey the ideal gas equation of state, $p_{\mathrm{n}}=\rho_{\mathrm{n}} R_{\mathrm{n}} T_{\mathrm{n}}$, where $p_{\mathrm{n}}$ is the static pressure of the neutral gas, $\rho_{\mathrm{n}}=m_{\mathrm{n}} n_{\mathrm{n}}$ is the neutral gas mass density, $n_{\mathrm{n}}$ is the neutral gas number density, $T_{\mathrm{n}}$ is the neutral gas temperature and $R_{\mathrm{n}}$ is the gas constant. Due to the low concentration of ions particles relative to the concentration of neutral molecules, ion-neutral 
inter-particle interactions have an insignificant influence on the motion of the neutral gas. It is therefore appropriate to assume that the neutral gas flow is unaffected by the ion particles. Accordingly, the well-known Navier-Stokes equations for a compressible fluid govern the transport of the neutral gas. For a two-dimensional axisymmetric coordinate system, the conservation form of these equations can be expressed as

$$
\frac{\partial \boldsymbol{U}_{\mathrm{n}}}{\partial t}+\frac{\partial \boldsymbol{F}_{\mathrm{n}}}{\partial z}+\frac{\partial \boldsymbol{G}_{\mathrm{n}}}{\partial r}=\frac{\partial \boldsymbol{F}_{\boldsymbol{v n}}}{\partial z}+\frac{\partial \boldsymbol{G}_{\boldsymbol{v} \mathrm{n}}}{\partial r}+\boldsymbol{S}_{\mathrm{an}},
$$

where $\boldsymbol{U}_{\mathrm{n}}$ is the neutral gas conserved variable solution vector given by

$$
\boldsymbol{U}_{\mathrm{n}}=\left[\begin{array}{llll}
\rho_{\mathrm{n}}, & \rho_{\mathrm{n}} u_{\mathrm{n}}, & \rho_{\mathrm{n}} v_{\mathrm{n}}, & \rho_{\mathrm{n}} \epsilon_{\mathrm{n}}
\end{array}\right]^{\mathrm{T}},
$$

$\boldsymbol{F}_{\mathrm{n}}$ and $\boldsymbol{G}_{\mathrm{n}}$ are the axial- and radial-direction inviscid flux vectors given by

$\boldsymbol{F}_{\mathrm{n}}=\left[\begin{array}{c}\rho_{\mathrm{n}} u_{\mathrm{n}} \\ \rho_{\mathrm{n}} u_{\mathrm{n}}^{2}+p_{\mathrm{n}} \\ \rho_{\mathrm{n}} u_{\mathrm{n}} v_{\mathrm{n}} \\ u_{\mathrm{n}}\left(\rho_{\mathrm{n}} \epsilon_{\mathrm{n}}+p_{\mathrm{n}}\right)\end{array}\right], \quad \boldsymbol{G}_{\mathrm{n}}=\left[\begin{array}{c}\rho_{\mathrm{n}} v_{\mathrm{n}} \\ \rho_{\mathrm{n}} u_{\mathrm{n}} v_{\mathrm{n}} \\ \rho_{\mathrm{n}} v_{\mathrm{n}}^{2}+p_{\mathrm{n}} \\ v_{\mathrm{n}}\left(\rho_{\mathrm{n}} \epsilon_{\mathrm{n}}+p_{\mathrm{n}}\right)\end{array}\right]$

and $\boldsymbol{F}_{\boldsymbol{v n}}$ and $\boldsymbol{G}_{\boldsymbol{v} \text { n }}$ are the axial- and radial-direction viscous flux vectors given by

$$
\begin{gathered}
\boldsymbol{F}_{\boldsymbol{v n}}=\left[\begin{array}{c}
0 \\
\tau_{\mathrm{n}_{z z}} \\
\tau_{\mathrm{n}_{r z}} \\
u_{\mathrm{n}} \tau_{\mathrm{n}_{z z}}+v_{\mathrm{n}} \tau_{\mathrm{n}_{r z}}-q_{\mathrm{n}_{z}}
\end{array}\right], \\
0 \\
\tau_{\mathrm{n}_{r z}} \\
\tau_{\mathrm{n}_{r r}} \\
\boldsymbol{G}_{\boldsymbol{v n}}=\left[\begin{array}{c} 
\\
u_{\mathrm{n}} \tau_{\mathrm{n}_{r z}}+v_{\mathrm{n}} \tau_{\mathrm{n}_{z z}}-q_{\mathrm{n}_{r}}
\end{array}\right] .
\end{gathered}
$$

The vector $\boldsymbol{S}_{\text {an }}$ contains source and viscous flux terms associated with the axisymmetric geometry and has the form

$$
\boldsymbol{S}_{\mathrm{an}}=\frac{1}{r}\left[\begin{array}{c}
-\rho_{\mathrm{n}} v_{\mathrm{n}} \\
-\rho_{\mathrm{n}} u_{\mathrm{n}} v_{\mathrm{n}}+\tau_{\mathrm{n}_{r z}} \\
-\rho_{\mathrm{n}} v_{\mathrm{n}}^{2}+\tau_{\mathrm{n}_{r r}}-\tau_{\mathrm{n}_{\theta \theta}} \\
-v_{\mathrm{n}}\left(\rho_{\mathrm{n}} \epsilon_{\mathrm{n}}+p_{\mathrm{n}}\right)+u_{\mathrm{n}} \tau_{\mathrm{n}_{r z}}+v_{\mathrm{n}} \tau_{\mathrm{n}_{r r}}-q_{\mathrm{n}_{r}}
\end{array}\right] .
$$

The variables $u_{\mathrm{n}}$ and $v_{\mathrm{n}}$ are the axial and radial components of the neutral gas velocity, $\boldsymbol{v}_{\mathrm{n}}$, respectively, and $\epsilon_{\mathrm{n}}=p_{\mathrm{n}} /$ $\left(\rho_{\mathrm{n}}\left(\gamma_{\mathrm{n}}-1\right)\right)+\left(u_{\mathrm{n}}^{2}+v_{\mathrm{n}}^{2}\right) / 2$ is the specific total energy. The components of the viscous stress tensor, $\tau_{\mathrm{n}}$, are given by

$$
\tau_{\mathrm{n}_{i j}}=\mu_{\mathrm{n}}\left(\frac{\partial v_{\mathrm{n}_{\mathrm{i}}}}{\partial x_{j}}+\frac{\partial v_{\mathrm{n}_{j}}}{\partial x_{i}}-\delta_{i j} \frac{2}{3} \nabla \cdot \boldsymbol{v}_{\mathrm{n}}\right),
$$

and the axial and radial components of the heat flux vector, $\boldsymbol{q}_{\mathrm{n}}$, follow from Fourier's law, $\boldsymbol{q}_{\mathrm{n}}=-\kappa_{\mathrm{n}} \boldsymbol{\nabla} T_{\mathrm{n}}$, where $\mu_{\mathrm{n}}$ is the dynamic viscosity, $\kappa_{\mathrm{n}}$ is the thermal conductivity and $\gamma_{\mathrm{n}}$ is the specific heat ratio for the neutral gas. Sutherland's law is used to prescribe the viscosity. Equation (13) is used here to model the high-speed flow of the neutral gas. The validity of the preceding equations for describing the high-speed neutral jet flows of interest here has been clearly demonstrated in previous studies by the authors [20].

\section{Numerical solution procedure}

\subsection{Solution of ion transport equations}

A parallel explicit higher-order Godunov-type finite-volume scheme is used to solve the five-moment ion transport equations given by equation (6) on multi-block quadrilateral meshes. The proposed scheme determines the ion motion given a precomputed neutral gas flow field and electric potential. In the finite-volume approach, the governing equations are integrated over quadrilateral cells of a structured multi-block quadrilateral mesh. This finite-volume formulation applied to cell $j$ is given by

$\frac{\mathrm{d} \boldsymbol{U}_{\mathrm{i}_{j}}}{\mathrm{~d} t}=-\frac{1}{A_{j}} \sum_{\text {faces }, k} \overrightarrow{\boldsymbol{F}}_{\mathrm{i}_{j k}} \cdot \overrightarrow{\boldsymbol{n}}_{\mathrm{j} k} \Delta \boldsymbol{\ell}_{j k}+\boldsymbol{S}_{\mathrm{ai}_{j}}+\boldsymbol{S}_{\mathrm{ei}_{j}}+\boldsymbol{S}_{\mathrm{ci}_{j}}$,

where $\overrightarrow{\boldsymbol{F}}_{\mathrm{i}}=\left(\boldsymbol{F}_{\mathrm{i}}, \boldsymbol{G}_{\mathrm{i}}\right), A_{j}$ is the area of cell $j$ and $\Delta \ell_{j k}$ and $\overrightarrow{\boldsymbol{n}}_{j k}$ are the length of the cell face $k$ and unit vector normal to the cell face or edge, respectively. The numerical fluxes at the faces of each cell are determined from the solution of a Riemann problem. Given the left and right solution states, $\boldsymbol{U}_{\mathrm{i}_{1}}$ and $\boldsymbol{U}_{\mathrm{i}_{\mathrm{r}}}$, at the cell interfaces, the numerical flux is given by

$$
\overrightarrow{\boldsymbol{F}}_{\mathrm{i}} \cdot \overrightarrow{\boldsymbol{n}}=\mathcal{F}_{\mathrm{i}}\left(\boldsymbol{U}_{\mathrm{i}_{1}}, \boldsymbol{U}_{\mathrm{i}_{\mathrm{r}}}, \boldsymbol{n}\right),
$$

where the numerical flux $\mathcal{F}_{\mathrm{i}}$ is evaluated by solving a Riemann problem in the direction defined by the normal to the face with initial data $\boldsymbol{U}_{1}$ and $\boldsymbol{U}_{\mathrm{r}}$. The left and right solution states are determined using the least-squares limited piecewise linear solution reconstruction procedure of Barth [21]. The modified limiter of Venkatakrishnan [22] has also been implemented. In the present algorithm, both exact and approximate Riemann solvers can be used to solve the Riemann problem. The Roe linearized Riemann solver [23], the HLLE-type flux function of Linde [24], the HLLC flux function [25] and the exact Riemann solver of Gottlieb and Groth [26] have all been implemented and may be used. For time-accurate calculations, predictor-corrector and fourth order RungeKutta time-marching methods are used to integrate the set of ordinary differential equations that result from this spatial discretization of the governing equations. The optimally smoothing multi-stage schemes developed by van Leer et al [27] are adopted for steady-state calculations. To cope with numerical stiffness, a semi-implicit treatment is used in the temporal discretization of the source terms associated with axisymmetric geometry, electric field and ion-neutral collisions.

Following the approach developed by Groth et al for computational magnetohydrodynamics [28, 29], a flexible block-based hierarchical data structure is used to maintain the connectivity of the quadrilateral solution blocks in the multiblock mesh and facilitate automatic solution-directed mesh adaptation according to physics-based refinement criteria. This data structure also lends itself naturally to domain decomposition and thereby enables efficient and scalable implementations of the algorithm on distributed-memory multi-processor architectures. 


\subsection{Solution of neutral flow equations}

A commercial computational fluid dynamics flow solver (CFD++, developed by Metacomp Technologies) is used to predict the high-speed expanding neutral gas flows. This flow solver employs an upwind total variation diminishing (TVD) finite-volume spatial discretization scheme in conjunction with a multigrid accelerated implicit time-marching procedure to solve the compressible axisymmetric form of the NavierStokes equations on unstructured triangular meshes [30,31]. A V-cycle and Gauss-Seidel smoother are employed in the multigrid convergence acceleration strategy. Clustering of the cells in the computational mesh may be used to help capture the important features of the complex neutral flows. The commercial solver also has parallel processing capabilities. The parallel implementation of the neutral gas solver has been developed using the MPI library [32] for performing interprocessor communications and the METIS graph partitioning software for performing a domain decomposition of the computational mesh [33]. The neutral flow solution is independent of the ion solution and can be obtained in a separate calculation. Linear interpolation is used to transfer the neutral flow solution from the unstructured triangular mesh used in the solution of the neutral flow equations to the multiblock quadrilateral mesh used by the finite-volume scheme for the ion transport equations.

\subsection{Modelling of electric field}

3.3.1. Dc electric potential. Like the neutral flow solution, the electric field is not dependent on the predicted ion solution and can also be determined in a separate independent computation. Assuming that the net charge density in the fluid volume of interest is negligible (i.e. although there is an excess of charge due to a lack of electrons or negative species, space charge effects can be neglected because of the low ion number densities), the stationary applied electric field, $\boldsymbol{E}$, satisfies Gauss' law $\boldsymbol{\nabla} \cdot \boldsymbol{E}=0$. By expressing the electric field in terms of an electric potential, $V$, such that $\boldsymbol{E}=-\nabla V$, the electric field can be determined by solving a Laplace equation for the electric potential. For a two-dimensional axisymmetric coordinate frame, the potential equation has the form

$$
\frac{\partial^{2} V}{\partial z^{2}}+\frac{\partial^{2} V}{\partial r^{2}}+\frac{1}{r} \frac{\partial V}{\partial r}=0 .
$$

An iterative solution procedure is used to solve equation (21) for $V$, subject to being appropriate for the applied voltages. This provides a complete description of the steady-state dc electric field.

\subsubsection{Pseudo-potential for rf quadrupole. In this study, the} traditional four-rod and novel hollow cylinder (with alternating electrode and insulator strips) configurations for rf quadrupoles are both considered. The rf field produced by the quadrupole is used to confine and focus the ions downstream of the skimmer into a narrow region near the axis of symmetry. By design, the rf field of the hollow cylinder is fully equivalent to the field created by the more traditional four-rod configuration [11] and in both cases a pseudo-potential formulation is used to prescribe the time-averaged electric field produced by the quadrupole. Although the pseudo-potential representation does not account for the micro-motion induced by the rf potential applied to the quadrupole, it does represent the timeaveraged influence of the field on the bulk motion of the ions which is of primary interest here.

The radial confinement of the ions in a quadrupole field can be described by the Mathieu equation [34,35]. The Mathieu equation has two types of solutions and can be characterized by the Mathieu parameter. The Mathieu parameter, $q$, is a measure of the stability of ions within the quadrupole and characterizes the maximum ion macro-motions permissible within the potential well produced by the time-averaged electric field. The Mathieu parameter can be expressed as $q=2 Q_{\mathrm{i}} U_{\mathrm{rf}} / m_{\mathrm{i}} \omega^{2} r_{0}^{2}$, where $\omega$ is the frequency of the applied rf field, $r_{0}$ is the radius of the hollow cylinder (the inscribed radius for conventional four-rod configurations) and $U_{\mathrm{rf}}$ is the peak-to-peak voltage of the rf field. If the rf voltage, $U_{\mathrm{rf}}$, is adjusted for each ion of a given mass, $m_{\mathrm{i}}$, such that the value of the Mathieu parameter, $q$, is fixed, the pseudo-potential or time-averaged effective potential can be expressed as follows:

$$
V_{\text {quad }}=\gamma_{\mathrm{rf}} \frac{q^{2} m_{\mathrm{i}} \omega^{2} r^{2}}{16 Q_{\mathrm{i}}}
$$

where $r$ is the radial distance from the axis of the quadrupole. Note that the effective potential can also be corrected by a term $\gamma_{\mathrm{rf}}$ reflecting the gas collisional latency effects on the rf focusing field [36], but has a negligible impact on the low pressure regimes considered here. The effective electric field is then given by the gradient of $V_{\text {quad }}$ and thus the radial and axial components of the pseudo-potential field within the rf cylinder are $E_{r}=-q^{2} m_{\mathrm{i}} \gamma_{\mathrm{rf}} \omega^{2} r / 8 Q_{\mathrm{i}}$ and $E_{z}=0$, respectively. From this, it should be quite evident that the electric field is expected to exert a confining/focusing effect on the ions. The pseudopotential field arising from the quadrupole is superimposed on the calculated dc potential solution and used directly in the calculation of the ion flow.

\section{Numerical results and discussion}

The mathematical formulation and solution algorithm outlined above have been used to perform numerical simulations of the transport of free ions through highly under-expanded jet flows of neutral gases within quadrupole interfaces of mass spectrometer systems. The simulations were carried out on a parallel cluster consisting of 4-way Hewlett-Packard ES40, ES45 and Integrity rx4640 servers with a total of 244 Alpha and Itanium 2 processors and 482 Gbytes of distributed memory. The results of the computations for axisymmetric neutral skimmer/quadrupole and pure quadrupole jets and ion flows under the action of dc and rf applied electric fields are now described.

\subsection{Neutral gas transport}

The neutral gas exhaustion through the orifice leading to the interface region of mass spectrometer systems with low 


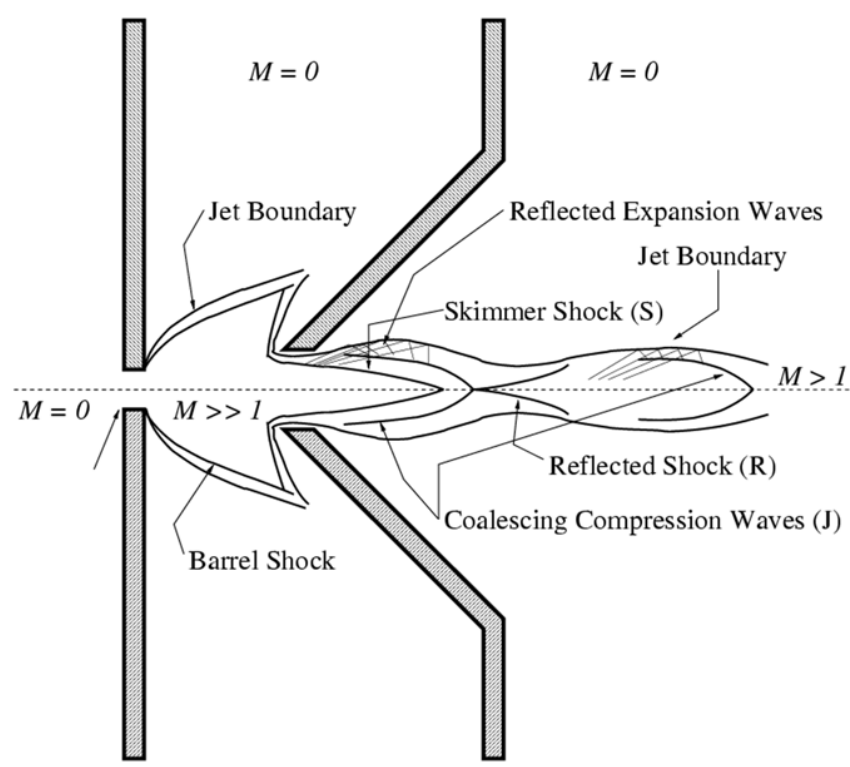

Figure 2. Schematic diagram of the skimmer jet flow shock structure showing the skimmer shock (S), the coalescing reflected compression waves from the jet boundary (J), the reflected shock (R) and a succession of expansion/re-compression cells (from [20])

background pressures undergoes a rapid expansion process and forms an under-expanded jet with high core flow Mach numbers. However, as discussed by Jugroot et al [20], the overall structure of the neutral jet with a gas skimmer present is quite different from that of a classical under-expanded free jet. The presence of the skimmer disrupts and prevents the formation of the free jet structure. The flow is deflected by the tip of the skimmer as the supersonic jet expands out from the orifice. A Mach disc does not form and the neutral gas remains highly supersonic in the core of the jet far downstream of the orifice and skimmer. The skimmer not only confines the jet expansion process but also acts to divide the neutral flow, diverting a significant portion of the neutral particles. Figure 2 provides a schematic diagram of the skimmer flow and shock structure, illustrating the essential flow features, including the skimmer shock and succession of expansion/re-compression cells occurring downstream of the skimmer.

In many MS interfaces, a quadrupole electric field is used to focus the ions downstream of the gas skimmer in preparation for a subsequent mass analysis. For the hollow cylinder rf quadrupole, the overall neutral flow is therefore further modified by the presence of the hollow cylinder as depicted in the predicted neutral flow solution of figure 3. For the case shown in the figure, a supersonic neutral gas emanates from an orifice of diameter, $d_{\mathrm{o}}=0.25 \mathrm{~mm}$, with an axisymmetric conical-shaped skimmer having a skimmer orifice diameter of $d_{\mathrm{s}}=2.5 \mathrm{~mm}$ and a cone angle of $\alpha_{\mathrm{s}}=60^{\circ}$. The tip of the skimmer is located $2 \mathrm{~mm}$ downstream of the orifice and a hollow cylinder rf quadrupole is located $19 \mathrm{~mm}$ away from the tip of the skimmer $(21 \mathrm{~mm}$ downstream of the orifice). Internal and external diameters of the cylinder are $8.4 \mathrm{~mm}$ and $9.0 \mathrm{~mm}$, respectively. The cross-section of the cylinder is more than 10 times that of the skimmer orifice. The numerical neutral solution was obtained with a 60000 -cell

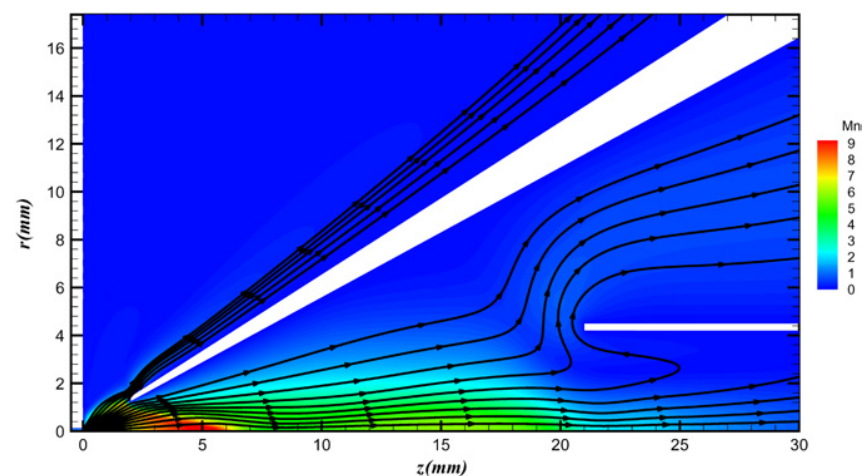

Figure 3. Neutral streamlines and distributions of the Mach number for a jet flow with an orifice diameter of $0.25 \mathrm{~mm}$, skimmer diameter of $2.5 \mathrm{~mm}$ and cylinder diameter of $8.4 \mathrm{~mm}$. The upstream pressure is 760 Torr and the pressure is 1 Torr and 5 mTorr downstream of the orifice and skimmer, respectively. (Colour online.)

Table 1. Physical properties of ions particles.

\begin{tabular}{llllll}
\hline Ion & $\mathrm{A}$ & $\mathrm{B}$ & $\mathrm{C}$ & $\mathrm{D}$ & $\mathrm{E}$ \\
\hline Mass (a.m.u) & 105 & 228 & 609 & 16951 & 692000 \\
Charge & +1 & +1 & +1 & +9 & +65 \\
$\sigma_{\mathrm{i}}\left(10^{-20} \mathrm{~m}^{2}\right)$ & 105 & 150 & 280 & 2560 & 19400 \\
$\gamma_{\mathrm{i}}$ & 1.2 & 1.1 & 1.1 & 1.05 & 1.05 \\
\hline
\end{tabular}

unstructured triangular mesh. Mesh clustering has been used to capture the important features of the flow.

The results of figure 3 indicate that, as expected, the upstream features of the neutral flow are not modified by the quadrupole cylinder; however, the effects of the cylinder on the flow are clearly visible downstream of the skimmer tip. The cylinder prevents the flow from further expanding freely, concentrating the flow towards the axis. The Mach number remains relatively high in the core of the jet $(M \approx 2-4)$ and then becomes subsonic near the entrance to the hollow cylinder. A stationary shock forms just upstream of the leading edge of the cylinder, which acts to deflect the oncoming supersonic neutral flow and produces a subsonic flow at the cylinder entrance. The flow through the cylinder is subsonic with $M \approx 0.2-0.3$.

\subsection{Ion transport in quadrupole interface}

The transport of ions through the neutral flow described above is now considered for several different ions with varying mass-to-charge ratios. The influences of the applied electric field (dc and rf) and neutral particles collisions are discussed. The numerical solutions for the ion flow were obtained using the parallel finite-volume method described previously with a total of 34 solution blocks and 100000 quadrilateral computational cells. Table 1 provides a summary of the ions considered. The five ions listed in the table are $\mathrm{A}$ (a generic low mass-to-charge ion); B (dodecyltrimethyl ammonium, $\mathrm{C}_{15} \mathrm{H}_{34} \mathrm{~N}$ ), C (a reserpine protonated molecule, $\mathrm{C}_{33} \mathrm{H}_{40} \mathrm{~N}_{2} \mathrm{O}_{9} . \mathrm{H}^{+}$), D (myoglobin-a protein) and $\mathrm{E}$ (proteasome $20 \mathrm{~S})$. 


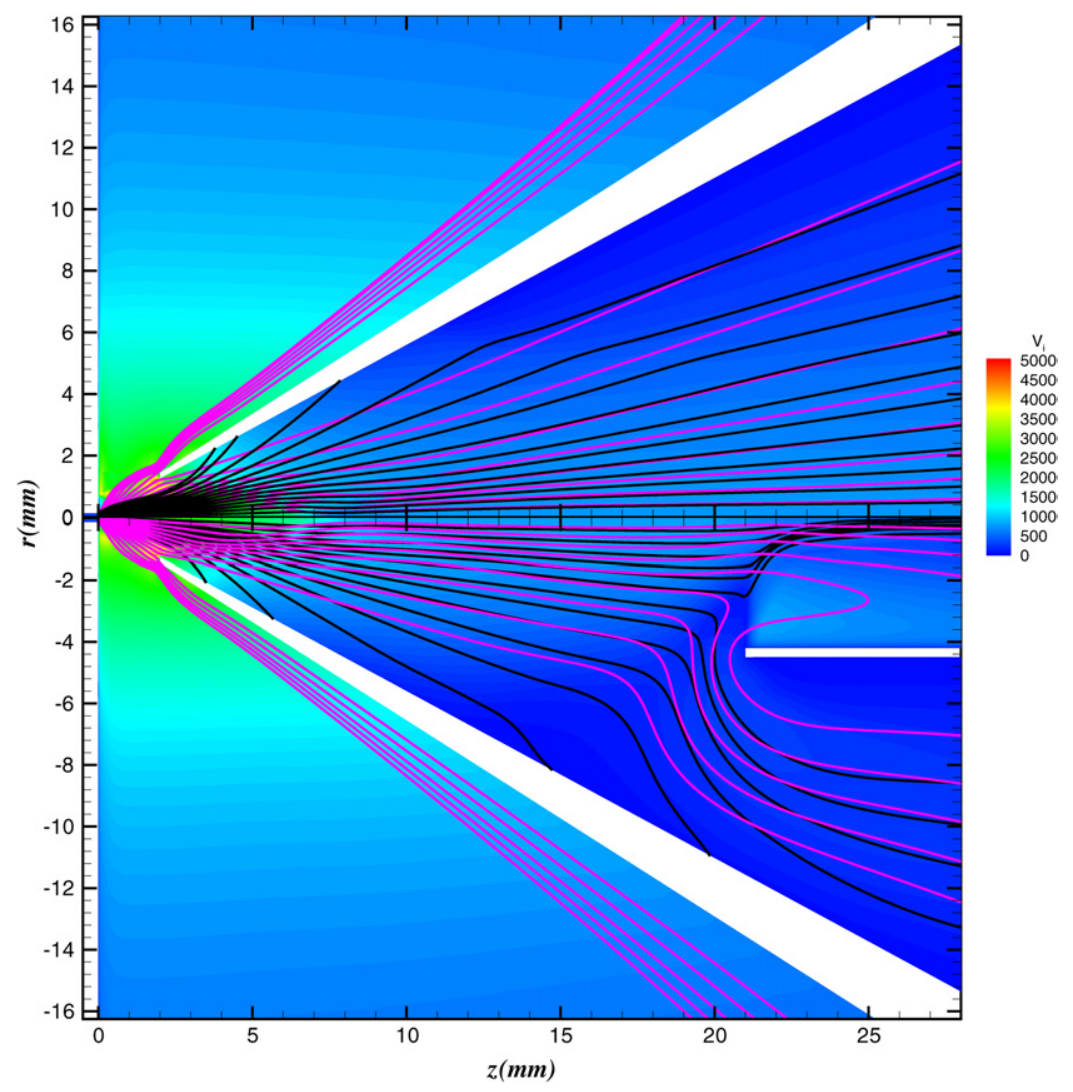

Figure 4. Computed ion particle (black) and neutral gas (purple) streamlines and distributions of the ion velocity ( $\mathrm{m} \mathrm{s}^{-1}$ ) for under-expanded skimmer jet flows. Results in upper and lower panels show ion B for an applied dc potential (without rf quadrupole) and a combined dc/rf potential (quadrupole with $q=0.2$ ), respectively. The potential on the orifice is $80 \mathrm{~V}$ and the skimmer is grounded. (Colour online.)

\subsubsection{Hollow cylindrical quadrupole. Figure 4 depicts} the trajectory of ion $\mathrm{B}$ and highlights the differences between an applied dc potential (orifice-skimmer only) and a superimposed $\mathrm{dc} / \mathrm{rf}$ potential for the quadrupole interface, as shown in the upper and lower panels. The effect of the rf potential within the cylinder is clearly evident as the ions are focused towards the axis of symmetry. The rf cylinder is in fact very efficient in focusing the ions. The mass flux of ions through the quadrupole is about $37 \%$ higher than that of the mass flux through the equivalent cross-section in the skimmeronly case.

Figure 5 compares the trajectories of ions $\mathrm{A}$ and $\mathrm{C}$ in the quadrupole interface as a result of the dc-rf superimposed field. For the results shown in the figure, the quadrupole pseudo-potential is the same for both ions with $q=0.2$ for ion $\mathrm{A}$ and $q=0.08$ for ion C. In general, the same global behaviour is observed for all the ions investigated. At the exit of the orifice, the ions follow the neutrals, but as they approach the skimmer regions, the ion trajectories clearly deviate from those of the neutrals due to the high electric field at the skimmer tip. Depending on the ion mass and charge, the ions exhibit a clear tendency to follow the diverging electric field lines and are strongly accelerated by the electric field. Joule heating and drag forces also lead to elevated ion temperatures in this region. Beyond the skimmer tip region, where the magnitude of the electric field gradually diminishes, all ions tend to be again governed by the neutral flow. Finally, the imposed pseudo-potential of the rf quadrupole produces a strong focusing of the ions towards the axis of symmetry. In fact, the rf field appears to be very efficient in confining the ions as depicted in figures 4 and 5.

Further optimization of the ion transmission efficiency is possible by tailoring the applied electric fields. This is demonstrated by the numerical results of figure 6 where enhanced focusing is achieved by applying a dc potential between the skimmer and the cylinder. The modified electric field lines redirect the ions towards the axis and remain focused downstream.

4.2.2. Traditional four-rod quadrupole. For comparison purposes, the traditional four-rod quadrupole has also been investigated. This interface consists of an orifice and a set of four rods downstream of the orifice. The atmospheric conditions are maintained in the reservoir upstream of the orifice and the background pressure in the quadrupole chamber is maintained at 2.3 Torr. The pressure is 5 mTorr at the exit aperture (into the next chamber) for the cases described herein. The predicted neutral gas and ion flow solutions are shown in figure 7 for this case. The figure depicts the solution in a vertical passing through the axis of symmetry for the quadrupole and lying in between the rods. The rods are not visible in the figure. Note that the neutral solutions for the four-rod quadrupole are inherently three-dimensional in nature; however, in the core region of the flow near the axis of 


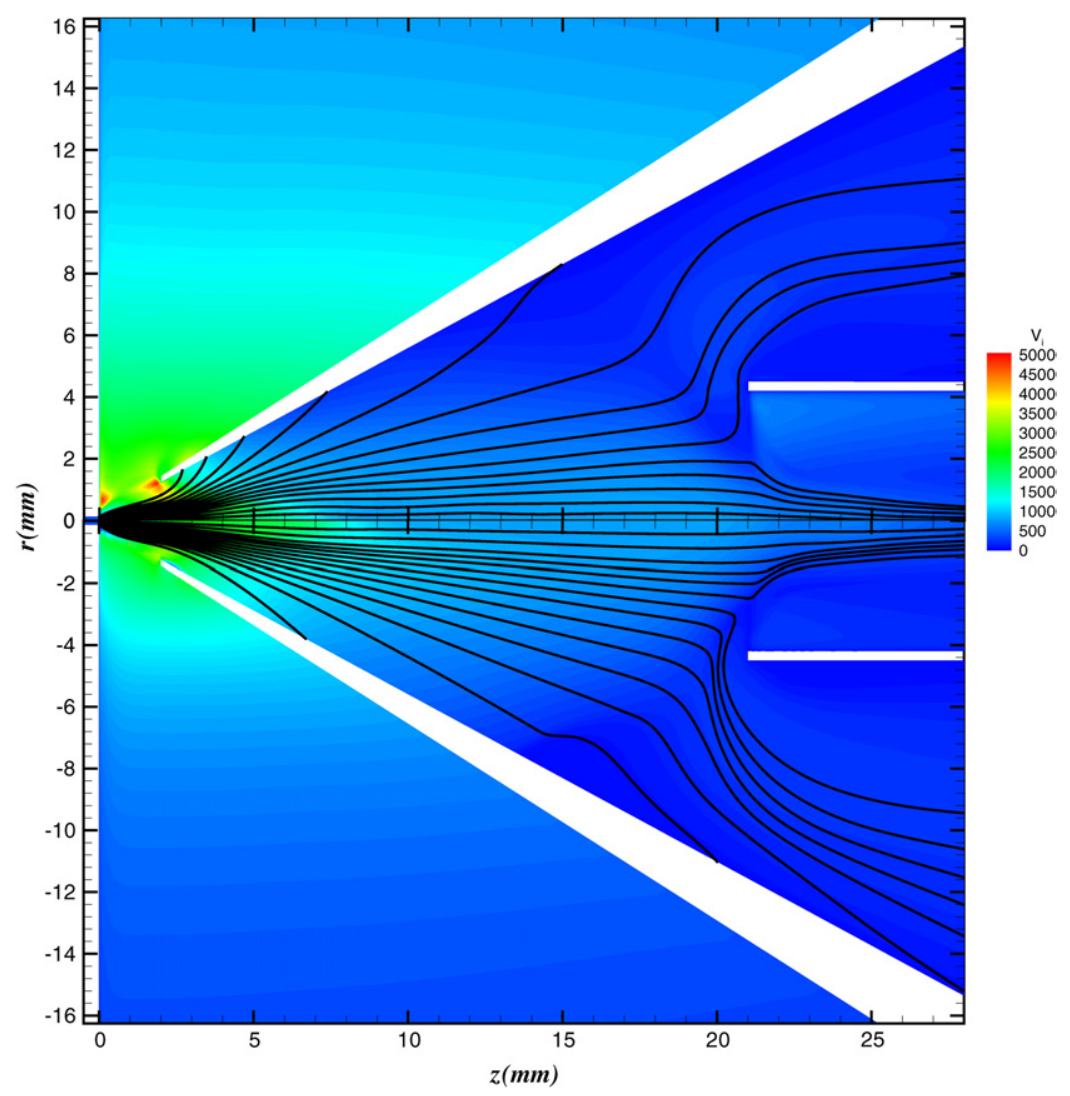

Figure 5. Computed ion particle (black) streamlines and distributions of the ion velocity $\left(\mathrm{m} \mathrm{s}^{-1}\right)$ for under-expanded skimmer jet flows. Results in upper and lower panels show two ions of different mass-to-charge ratios (ions A and C), respectively, for a combined dc/rf potential ( $q=0.2$ for ion A and $q=0.08$ for ion C). (Colour online.)

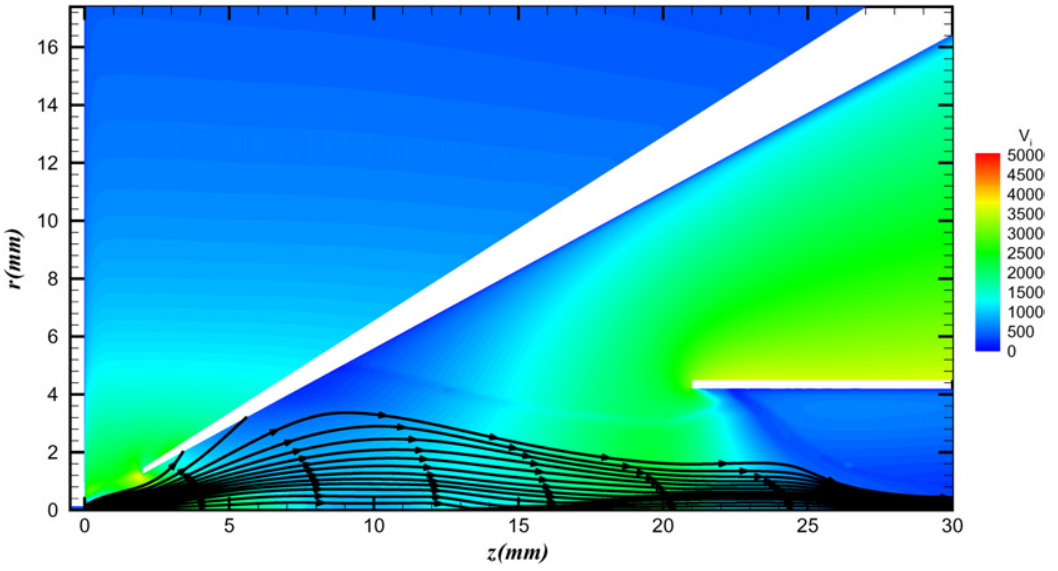

Figure 6. Computed ion particle B (black) streamlines and distributions of the ion velocity $\left(\mathrm{m} \mathrm{s}^{-1}\right)$. The potential on the orifice is $80 \mathrm{~V}$ and the skimmer is maintained at $20 \mathrm{~V}$ while the external cylinder layer is grounded; the Mathieu parameter for the quadrupole pseudo-potential is $q=0.2$. (Colour online.)

symmetry which is of particular interest for the ion trajectory simulations, an axisymmetric representation is reasonable and is therefore used as a first approximation. A projection of the three-dimensional solution on to a plane shown in figure 7 is used for the calculation of the ion-neutral coupling. Likewise an axisymmetric representation of the electric fields is used in these simulations.

The predicted neutral flow solution shown in the upper panel of figure 7 indicates that the flow impacts the tip of the rods upstream as the supersonic jet expands out from the orifice. The neutral flow is partially diverted by the rods but a significant portion of the neutral gas however remains well focused within the rods further downstream as indicated by the streamlines which are aligned with the rods in the core of the flow. The predicted ion motion corresponding to this projected neutral flow solution is shown in the lower panel of figure 7 . The ion streamlines indicate significant focusing of the charged species towards the axis of symmetry after the initial losses near the entrance to the quadrupole. Downstream of the rod entrance, the confining effect of the rf voltage is well observed 


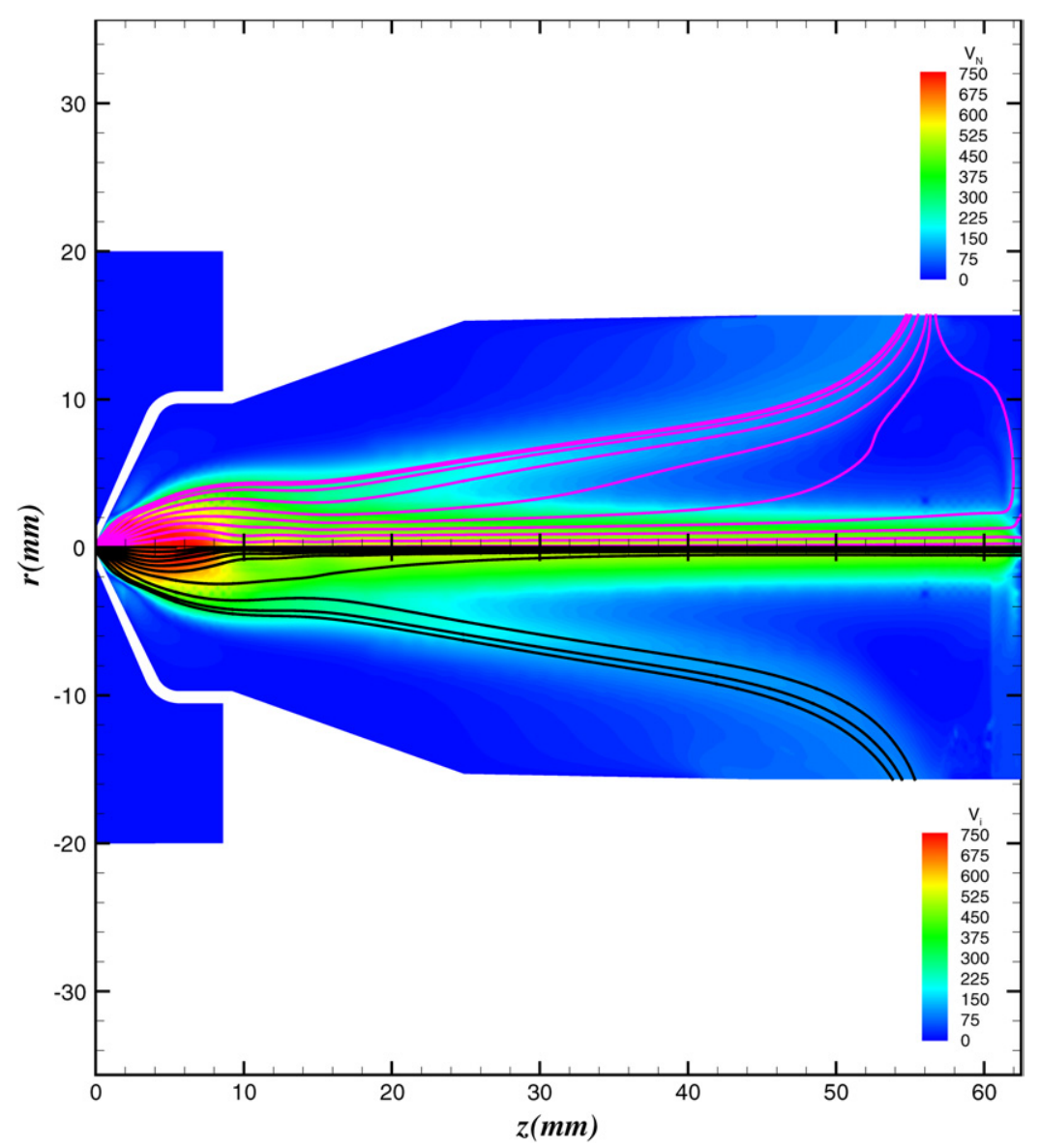

Figure 7. Computed ion particle (black) and neutral gas (purple) streamlines and distributions of the ion and neutral velocity ( $\mathrm{m} \mathrm{s}^{-1}$ ) for quadrupole jet flows. Results show ion B for $q=0.2$. The orifice diameter is $0.6 \mathrm{~mm}$ and the inter-electrode distance is $4 \mathrm{~mm}$; the tip of the rod is $2 \mathrm{~mm}$ away from the orifice. (Colour online.)

in the ion motion and prevents the ions from diffusing far from the axis of symmetry. In quantitative terms, the neutral flux through the exit aperture (as compared with the flow through the orifice) is $10.7 \%$, whereas the corresponding ion flux is $66 \%$ for the case presented, clearly indicating the benefit of the quadrupole field. Some ion loss is observed in the exit aperture region as a result of entrainment by the neutral gas, but this loss is not significant. To summarize, due to neutral/ion collisions, the neutral gas dynamics has a localized but strong effect on the ion trajectories, especially in the supersonic jet upstream and at the entrance of the quadrupole, whereas the electric field has a more gradual influence on the ions, as illustrated by the gradual confinement of the ion streamlines. Globally, these two effects can be combined to control ion transport through the interfaces of mass spectrometer systems.

\section{Concluding remarks}

The transport of free ions through highly under-expanded jet flows of neutral gases and in the presence of applied electric $\mathrm{dc}$ and rf fields has been investigated using continuum-based (fluid) numerical simulations. A five-moment mathematical model and parallel multi-block numerical solution procedure have been developed and described for predicting the ion transport. The model incorporates the effects of ion-neutral collision processes and is used in conjunction with a NavierStokes flow solver for the neutral gas to examine the key features of the ion motion. The combined effect of the applied (dc and rf) electric field and neutral collision processes with the diluted background gas results in a strong tendency for ion focusing towards the axis of symmetry, with the overall efficiency of the focusing being governed by the massto-charge ratio. The neutral gas dynamics has a strong influence on the ion transport whereas the electric field imparts a more gradual effect. Future work will involve extending the modelling to three-dimensional flow geometries and investigating the application of high-order moment models to describe non-equilibrium ion transport phenomena.

\section{Acknowledgments}

This research was supported by the Natural Sciences and Engineering Research Council of Canada (NSERC CRD Grant 254794-01). Funding for the parallel computing facility used to perform the numerical simulations described herein was obtained from the Canadian Foundation for Innovation and Ontario Innovation Trust (CFI Project No 2169). The authors are very grateful to these funding agencies for this support. 


\section{References}

[1] Groves J and Wadley H 1997 Composites B 28 57-69

[2] Martinez-Sanchez M and Pollard J E 1998 J. Propulsion Power 14 688-99

[3] Jugroot M and Harvey J K 2001 Aeronaut. J. 105 613-18

[4] Jugroot M, Bayle P, Yousfi M and Eichwald O 1999 J. Phys. D: Appl. Phys. 32 106-20

[5] Cinalli M and Keppens R 2006 J. Phys. D: Appl. Phys. 39 4589-600

[6] Pal S, Dey S and Miebach 2007 J. Phys. D: Appl. Phys. 40 3128-36

[7] Cravens T E 1997 Physics of Solar System Plasmas (Cambridge, UK: Cambridge University Press)

[8] Thomas R 2001 Spectroscopy 16 28-37

[9] Furuya H, Fujimaki S, Kambara S, Suzuki S, Hashimoto Y, Okazaki S, Wada A, Beech I, Sunner J and Hiraoka K 2005 Rapid Commun. Mass Spectrom. 19 2433-42

[10] Glish G L and Vachet R W 2003 Nature Rev. Drug Discovery 2 140-50

[11] Prestage J 1999 NASA Tech. Brief NPO-20011

[12] Jugroot M, Groth C P T, Thomson B, Baranov V and Collings B A 2003 Paper AIAA-2003-4210

[13] Jugroot M, Groth C P T, Thomson B, Baranov V and Collings B A 2004 J. Phys. D: Appl. Phys. 37 550-9

[14] Burgers J M 1969 Flow Equations for Composite Gases (New York: Academic)

[15] Barakat A R and Schunk R W 1982 Plasma Phys. 24 389-418

[16] Gombosi T I and Rasmussen C E 1991 J. Geophys. Res. 96 7759-78

[17] Gombosi T I 1994 Gaskinetic Theory (Cambridge: Cambridge University Press)
[18] Bhatnagar P L, Gross E P and Krook M 1954 Phys. Rev. 94 511-25

[19] Clemmer D E and Jarrold M F 1997 J. Mass Spectrom. 32 577-92

[20] Jugroot M, Groth C P T, Thomson B, Baranov V and Collings B A 2004 J. Phys. D: Appl. Phys. 37 1289-300

[21] Barth T J 1993 Paper AIAA-93-0668

[22] Venkatakrishnan V 1993 Paper AIAA-93-0880

[23] Roe P L 1981 J. Comput. Phys. 43 357-72

[24] Linde T J 1998 PhD Thesis University of Michigan

[25] Toro E F, Spruce M and Speares W 1994 Shock Waves 4 25-34

[26] Gottlieb J J and Groth C P T 1988 J. Comput. Phys. 78 437-58

[27] van Leer B, Tai C H and Powell K G 1989 Paper AIAA-89-1933-CP

[28] Groth C P T, Zeeuw D L D, Powell K G, Gombosi T I and Stout Q F 1999 Paper AIAA-99-3273

[29] Groth C P T, De Zeeuw D L, Gombosi T I and Powell K G 2000 J. Geophys. Res. 105 25053-78

[30] Peroomian O, Chakravarthy S and Goldberg U C 1997 Paper AIAA-97-0724

[31] Peroomian O, Chakravarthy S and Goldberg U C 1998 Paper AIAA-98-0116

[32] Gropp W, Lusk E and Skjellum A 1999 Using MPI (Cambridge, MA: MIT Press)

[33] Karypis G and Kumar V 1998 J. Parallel Distrib. Comput. 48 96-129

[34] Paul W 1990 Rev. Mod. Phys. 62 531-40

[35] Leibfried D, Blatt R, Monroe C and Wineland D 2003 Rev. Mod. Phys. 75 281-324

[36] Tolmachev A V, Chernushevish I V, Dodonov A F and Standing K G 1997 Nucl. Instrum. Methods Phys. Res. B $124112-19$ 